\title{
Diagnosis and Classification of Diabetes Mellitus
}

American Diabetes Association

\section{DEFINITION AND DESCRIPTION OF DIABETES} MELLITUS-Diabetes is a group of metabolic diseases characterized by hyperglycemia resulting from defects in insulin secretion, insulin action, or both. The chronic hyperglycemia of diabetes is associated with long-term damage, dysfunction, and failure of different organs, especially the eyes, kidneys, nerves, heart, and blood vessels.

Several pathogenic processes are involved in the development of diabetes. These range from autoimmune destruction of the $\beta$-cells of the pancreas with consequent insulin deficiency to abnormalities that result in resistance to insulin action. The basis of the abnormalities in carbohydrate, fat, and protein metabolism in diabetes is deficient action of insulin on target tissues. Deficient insulin action results from inadequate insulin secretion and/or diminished tissue responses to insulin at one or more points in the complex pathways of hormone action. Impairment of insulin secretion and defects in insulin action frequently coexist in the same patient, and it is often unclear which abnormality, if either alone, is the primary cause of the hyperglycemia.

Symptoms of marked hyperglycemia include polyuria, polydipsia, weight loss, sometimes with polyphagia, and blurred vision. Impairment of growth and susceptibility to certain infections may also accompany chronic hyperglycemia. Acute, life-threatening consequences of uncontrolled diabetes are hyperglycemia with ketoacidosis or the nonketotic hyperosmolar syndrome.

Long-term complications of diabetes include retinopathy with potential loss of vision; nephropathy leading to renal failure; peripheral neuropathy with risk of foot ulcers, amputations, and Charcot joints; and autonomic neuropathy causing gastrointestinal, genitourinary, and cardiovascular symptoms and sexual dysfunction. Patients with diabetes have an increased incidence of atherosclerotic cardiovascular, peripheral arterial, and cerebrovascular disease. Hypertension and abnormalities of lipoprotein metabolism are often found in people with diabetes.

The vast majority of cases of diabetes fall into two broad etiopathogenetic categories (discussed in greater detail below). In one category, type 1 diabetes, the cause is an absolute deficiency of insulin secretion. Individuals at increased risk of developing this type of diabetes can often be identified by serological evidence of an autoimmune pathologic process occurring in the pancreatic islets and by genetic markers. In the other, much more prevalent category, type 2 diabetes, the cause is a combination of resistance to insulin action and an inadequate compensatory insulin secretory response. In the latter category, a degree of hyperglycemia sufficient to cause pathologic and functional changes in various target tissues, but without clinical symptoms, may be present for a long period of time before diabetes is detected. During this asymptomatic period, it is possible to demonstrate an abnormality in carbohydrate metabolism by measurement of plasma glucose in the fasting state or after a challenge with an oral glucose load or by AlC.

The degree of hyperglycemia (if any) may change over time, depending on the extent of the underlying disease process (Fig. 1). A disease process may be present but may not have progressed far enough to cause hyperglycemia. The same disease process can cause impaired fasting glucose (IFG) and/or impaired glucose tolerance (IGT) without fulfilling the criteria for the diagnosis of diabetes. In some individuals with diabetes, adequate glycemic control can be achieved with weight reduction, exercise, and/or oral glucose-lowering

Updated Fall 2012

DOI: $10.2337 / \mathrm{dcl} 13-5067$

(C) 2013 by the American Diabetes Association. Readers may use this article as long as the work is properly cited, the use is educational and not for profit, and the work is not altered. See http://creativecommons.org/ licenses/by-nc-nd/3.0/ for details.

agents. These individuals therefore do not require insulin. Other individuals who have some residual insulin secretion but require exogenous insulin for adequate glycemic control can survive without it. Individuals with extensive $\beta$-cell destruction and therefore no residual insulin secretion require insulin for survival. The severity of the metabolic abnormality can progress, regress, or stay the same. Thus, the degree of hyperglycemia reflects the severity of the underlying metabolic process and its treatment more than the nature of the process itself.

\section{CLASSIFICATION OF DIABETES MELLITUS AND OTHER CATECORIES Of GLUcose}

RECULATION-Assigning a type of diabetes to an individual often depends on the circumstances present at the time of diagnosis, and many diabetic individuals do not easily fit into a single class. For example, a person diagnosed with gestational diabetes mellitus (GDM) may continue to be hyperglycemic after delivery and may be determined to have, in fact, type 2 diabetes. Alternatively, a person who acquires diabetes because of large doses of exogenous steroids may become normoglycemic once the glucocorticoids are discontinued, but then may develop diabetes many years later after recurrent episodes of pancreatitis. Another example would be a person treated with thiazides who develops diabetes years later. Because thiazides in themselves seldom cause severe hyperglycemia, such individuals probably have type 2 diabetes that is exacerbated by the drug. Thus, for the clinician and patient, it is less important to label the particular type of diabetes than it is to understand the pathogenesis of the hyperglycemia and to treat it effectively.

Type 1 diabetes ( $\beta$-cell destruction, usually leading to absolute insulin deficiency)

Immune-mediated diabetes. This form of diabetes, which accounts for only $5-10 \%$ of those with diabetes, previously encompassed by the terms insulindependent diabetes or juvenile-onset diabetes, results from a cellular-mediated autoimmune destruction of the $\beta$-cells of 


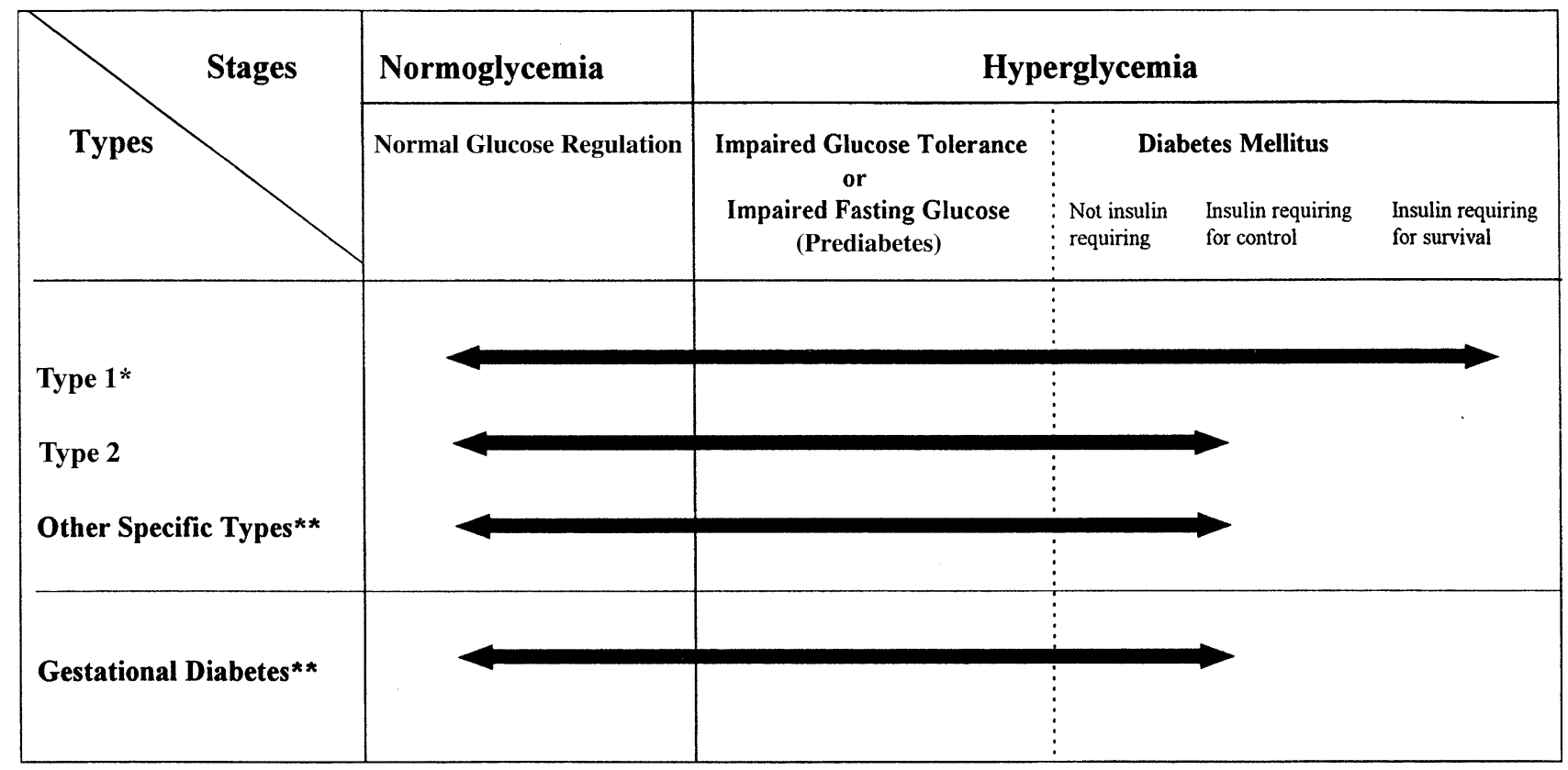

Figure 1-Disorders of glycemia: etiologic types and stages. *Even after presenting in ketoacidosis, these patients can briefly return to normoglycemia without requiring continuous therapy (i.e., "honeymoon" remission); **in rare instances, patients in these categories (e.g., Vacor toxicity, type 1 diabetes presenting in pregnancy) may require insulin for survival.

the pancreas. Markers of the immune destruction of the $\beta$-cell include islet cell autoantibodies, autoantibodies to insulin, autoantibodies to GAD (GAD65), and autoantibodies to the tyrosine phosphatases IA-2 and IA-2 $\beta$. One and usually more of these autoantibodies are present in 85$90 \%$ of individuals when fasting hyperglycemia is initially detected. Also, the disease has strong HLA associations, with linkage to the DQA and DQB genes, and it is influenced by the DRB genes. These HLA-DR/DQ alleles can be either predisposing or protective.

In this form of diabetes, the rate of $\beta$-cell destruction is quite variable, being rapid in some individuals (mainly infants and children) and slow in others (mainly adults). Some patients, particularly children and adolescents, may present with ketoacidosis as the first manifestation of the disease. Others have modest fasting hyperglycemia that can rapidly change to severe hyperglycemia and/or ketoacidosis in the presence of infection or other stress. Still others, particularly adults, may retain residual $\beta$-cell function sufficient to prevent ketoacidosis for many years; such individuals eventually become dependent on insulin for survival and are at risk for ketoacidosis. At this latter stage of the disease, there is little or no insulin secretion, as manifested by low or undetectable levels of plasma C-peptide. Immune-mediated diabetes commonly occurs in childhood and adolescence, but it can occur at any age, even in the 8th and 9th decades of life.

Autoimmune destruction of $\beta$-cells has multiple genetic predispositions and is also related to environmental factors that are still poorly defined. Although patients are rarely obese when they present with this type of diabetes, the presence of obesity is not incompatible with the diagnosis. These patients are also prone to other autoimmune disorders such as Graves' disease, Hashimoto's thyroiditis, Addison's disease, vitiligo, celiac sprue, autoimmune hepatitis, myasthenia gravis, and pernicious anemia.

Idiopathic diabetes. Some forms of type 1 diabetes have no known etiologies. Some of these patients have permanent insulinopenia and are prone to ketoacidosis, but have no evidence of autoimmunity. Although only a minority of patients with type 1 diabetes fall into this category, of those who do, most are of African or Asian ancestry. Individuals with this form of diabetes suffer from episodic ketoacidosis and exhibit varying degrees of insulin deficiency between episodes. This form of diabetes is strongly inherited, lacks immunological evidence for $\beta$-cell autoimmunity, and is not HLA associated. An absolute requirement for insulin replacement therapy in affected patients may come and go.
Type 2 diabetes (ranging from predominantly insulin resistance with relative insulin deficiency to predominantly an insulin secretory defect with insulin resistance)

This form of diabetes, which accounts for 90-95\% of those with diabetes, previously referred to as non-insulin-dependent diabetes, type 2 diabetes, or adultonset diabetes, encompasses individuals who have insulin resistance and usually have relative (rather than absolute) insulin deficiency At least initially, and often throughout their lifetime, these individuals do not need insulin treatment to survive. There are probably many different causes of this form of diabetes. Although the specific etiologies are not known, autoimmune destruction of $\beta$-cells does not occur, and patients do not have any of the other causes of diabetes listed above or below.

Most patients with this form of diabetes are obese, and obesity itself causes some degree of insulin resistance. Patients who are not obese by traditional weight criteria may have an increased percentage of body fat distributed predominantly in the abdominal region. Ketoacidosis seldom occurs spontaneously in this type of diabetes; when seen, it usually arises in association with the stress of another illness such as infection. This form of diabetes frequently goes undiagnosed for many years because the hyperglycemia 
develops gradually and at earlier stages is often not severe enough for the patient to notice any of the classic symptoms of diabetes. Nevertheless, such patients are at increased risk of developing macrovascular and microvascular complications. Whereas patients with this form of diabetes may have insulin levels that appear normal or elevated, the higher blood glucose levels in these diabetic patients would be expected to result in even higher insulin values had their $\beta$-cell function been normal. Thus, insulin secretion is defective in these patients and insufficient to compensate for insulin resistance. Insulin resistance may improve with weight reduction and/or pharmacological treatment of hyperglycemia but is seldom restored to normal. The risk of developing this form of diabetes increases with age, obesity, and lack of physical activity. It occurs more frequently in women with prior GDM and in individuals with hypertension or dyslipidemia, and its frequency varies in different racial/ethnic subgroups. It is often associated with a strong genetic predisposition, more so than is the autoimmune form of type 1 diabetes. However, the genetics of this form of diabetes are complex and not fully defined.

\section{Other specific types of diabetes}

Genetic defects of the $\boldsymbol{\beta}$-cell. Several forms of diabetes are associated with monogenetic defects in $\beta$-cell function. These forms of diabetes are frequently characterized by onset of hyperglycemia at an early age (generally before age 25 years). They are referred to as maturityonset diabetes of the young (MODY) and are characterized by impaired insulin secretion with minimal or no defects in insulin action. They are inherited in an autosomal dominant pattern. Abnormalities at six genetic loci on different chromosomes have been identified to date. The most common form is associated with mutations on chromosome 12 in a hepatic transcription factor referred to as hepatocyte nuclear factor (HNF)-1 $\alpha$. A second form is associated with mutations in the glucokinase gene on chromosome $7 \mathrm{p}$ and results in a defective glucokinase molecule. Glucokinase converts glucose to glucose-6-phosphate, the metabolism of which, in turn, stimulates insulin secretion by the $\beta$-cell. Thus, glucokinase serves as the "glucose sensor" for the $\beta$-cell. Because of defects in the glucokinase gene, increased plasma levels of glucose are necessary to elicit normal levels of insulin secretion. The less common forms result from mutations in other transcription factors, including $\mathrm{HNF}-4 \alpha$, HNF-1 $\beta$, insulin promoter factor (IPF)1 , and NeuroD1.

Diabetes diagnosed in the first 6 months of life has been shown not to be typical autoimmune type 1 diabetes. This so-called neonatal diabetes can either be transient or permanent. The most common genetic defect causing transient disease is a defect on ZAC/HYAMI imprinting, whereas permanent neonatal diabetes is most commonly a defect in the gene encoding the Kir6.2 subunit of the $\beta$-cell $K_{\text {ATP }}$ channel. Diagnosing the latter has implications, since such children can be well managed with sulfonylureas.

Point mutations in mitochondrial DNA have been found to be associated with diabetes and deafness The most common mutation occurs at position 3,243 in the tRNA leucine gene, leading to an A-to-G transition. An identical lesion occurs in the MELAS syndrome (mitochondrial myopathy, encephalopathy, lactic acidosis, and stroke-like syndrome); however, diabetes is not part of this syndrome, suggesting different phenotypic expressions of this genetic lesion.

Genetic abnormalities that result in the inability to convert proinsulin to insulin have been identified in a few families, and such traits are inherited in an autosomal dominant pattern. The resultant glucose intolerance is mild. Similarly, the production of mutant insulin molecules with resultant impaired receptor binding has also been identified in a few families and is associated with an autosomal inheritance and only mildly impaired or even normal glucose metabolism.

Genetic defects in insulin action. There are unusual causes of diabetes that result from genetically determined abnormalities of insulin action. The metabolic abnormalities associated with mutations of the insulin receptor may range from hyperinsulinemia and modest hyperglycemia to severe diabetes. Some individuals with these mutations may have acanthosis nigricans. Women may be virilized and have enlarged, cystic ovaries. In the past, this syndrome was termed type A insulin resistance. Leprechaunism and the RabsonMendenhall syndrome are two pediatric syndromes that have mutations in the insulin receptor gene with subsequent alterations in insulin receptor function and extreme insulin resistance. The former has characteristic facial features and is usually fatal in infancy, while the latter is associated with abnormalities of teeth and nails and pineal gland hyperplasia.

Alterations in the structure and function of the insulin receptor cannot be demonstrated in patients with insulinresistant lipoatrophic diabetes. Therefore, it is assumed that the lesion(s) must reside in the postreceptor signal transduction pathways.

Diseases of the exocrine pancreas. Any process that diffusely injures the pancreas can cause diabetes. Acquired processes include pancreatitis, trauma, infection, pancreatectomy, and pancreatic carcinoma. With the exception of that caused by cancer, damage to the pancreas must be extensive for diabetes to occur; adrenocarcinomas that involve only a small portion of the pancreas have been associated with diabetes. This implies a mechanism other than simple reduction in $\beta$-cell mass. If extensive enough, cystic fibrosis and hemochromatosis will also damage $\beta$-cells and impair insulin secretion. Fibrocalculous pancreatopathy may be accompanied by abdominal pain radiating to the back and pancreatic calcifications identified on X-ray examination. Pancreatic fibrosis and calcium stones in the exocrine ducts have been found at autopsy.

Endocrinopathies. Several hormones (e.g., growth hormone, cortisol, glucagon, epinephrine) antagonize insulin action. Excess amounts of these hormones (e.g., acromegaly, Cushing's syndrome, glucagonoma, pheochromocytoma, respectively) can cause diabetes. This generally occurs in individuals with preexisting defects in insulin secretion, and hyperglycemia typically resolves when the hormone excess is resolved.

Somatostatinomas, and aldosteronoma-induced hypokalemia, can cause diabetes, at least in part, by inhibiting insulin secretion. Hyperglycemia generally resolves after successful removal of the tumor.

Drug- or chemical-induced diabetes. Many drugs can impair insulin secretion. These drugs may not cause diabetes by themselves, but they may precipitate diabetes in individuals with insulin resistance. In such cases, the classification is unclear because the sequence or relative importance of $\beta$-cell dysfunction and insulin resistance is unknown. Certain toxins such as Vacor (a rat poison) and intravenous pentamidine can permanently destroy pancreatic $\beta$-cells. Such drug reactions fortunately are rare. There are also many drugs and hormones that 
can impair insulin action. Examples include nicotinic acid and glucocorticoids. Patients receiving $\alpha$-interferon have been reported to develop diabetes associated with islet cell antibodies and, in certain instances, severe insulin deficiency. The list shown in Table 1 is not all-inclusive, but reflects the more commonly recognized drug-, hormone-, or toxin-induced forms of diabetes.

Infections. Certain viruses have been associated with $\beta$-cell destruction. Diabetes occurs in patients with congenital rubella, although most of these patients have HLA and immune markers characteristic of type 1 diabetes. In addition, coxsackievirus B, cytomegalovirus, adenovirus, and mumps have been implicated in inducing certain cases of the disease.

Uncommon forms of immune-mediated diabetes. In this category, there are two known conditions, and others are likely to occur. The stiff-man syndrome is an autoimmune disorder of the central nervous system characterized by stiffness of the axial muscles with painful spasms. Patients usually have high titers of the GAD autoantibodies, and approximately one-third will develop diabetes.

Anti-insulin receptor antibodies can cause diabetes by binding to the insulin receptor, thereby blocking the binding of insulin to its receptor in target tissues. However, in some cases, these antibodies can act as an insulin agonist after binding to the receptor and can thereby cause hypoglycemia. Anti-insulin receptor antibodies are occasionally found in patients with systemic lupus erythematosus and other autoimmune diseases. As in other states of extreme insulin resistance, patients with anti-insulin receptor antibodies often have acanthosis nigricans. In the past, this syndrome was termed type B insulin resistance. Other genetic syndromes sometimes associated with diabetes. Many genetic syndromes are accompanied by an increased incidence of diabetes. These include the chromosomal abnormalities of Down syndrome, Klinefelter syndrome, and Turner syndrome. Wolfram syndrome is an autosomal recessive disorder characterized by insulin-deficient diabetes and the absence of $\beta$-cells at autopsy. Additional manifestations include diabetes insipidus, hypogonadism, optic atrophy, and neural deafness. Other syndromes are listed in Table 1.

\section{GDM}

For many years, GDM has been defined as any degree of glucose intolerance with
Table 1-Etiologic classification of diabetes mellitus

I. Type 1 diabetes ( $\beta$-cell destruction, usually leading to absolute insulin deficiency)

A. Immune mediated

B. Idiopathic

II. Type 2 diabetes (may range from predominantly insulin resistance with relative insulin deficiency to a predominantly secretory defect with insulin resistance)

III. Other specific types

A. Genetic defects of $\beta$-cell function

1. MODY 3 (Chromosome 12, HNF-1 $\alpha$ )

2. MODY 1 (Chromosome 20, HNF-4 $\alpha$ )

3. MODY 2 (Chromosome 7, glucokinase)

4. Other very rare forms of MODY (e.g., MODY 4: Chromosome 13, insulin promoter factor-1; MODY 6: Chromosome 2, NeuroD1; MODY 7: Chromosome 9, carboxyl ester lipase)

5. Transient neonatal diabetes (most commonly ZAC/HYAMI imprinting defect on 6q24)

6. Permanent neonatal diabetes (most commonly KCNJ1l gene encoding Kir6.2 subunit of $\beta$-cell $\mathrm{K}_{\mathrm{ATP}}$ channel)

7. Mitochondrial DNA

8. Others

B. Genetic defects in insulin action

1. Type A insulin resistance

2. Leprechaunism

3. Rabson-Mendenhall syndrome

4. Lipoatrophic diabetes

5. Others

C. Diseases of the exocrine pancreas

1. Pancreatitis

2. Trauma/pancreatectomy

3. Neoplasia

4. Cystic fibrosis

5. Hemochromatosis

6. Fibrocalculous pancreatopathy

7. Others

D. Endocrinopathies

1. Acromegaly

2. Cushing's syndrome

3. Glucagonoma

4. Pheochromocytoma

5. Hyperthyroidism

6. Somatostatinoma

7. Aldosteronoma

8. Others

E. Drug or chemical induced

1. Vacor

2. Pentamidine

3. Nicotinic acid

4. Glucocorticoids

5. Thyroid hormone

6. Diazoxide

7. $\beta$-Adrenergic agonists

8. Thiazides

9. Dilantin

10. $\gamma$-Interferon

11. Others

F. Infections

1. Congenital rubella

2. Cytomegalovirus

3. Others

G. Uncommon forms of immune-mediated diabetes

1. "Stiff-man" syndrome

2. Anti-insulin receptor antibodies

3. Others

H. Other genetic syndromes sometimes associated with diabetes

1. Down syndrome

2. Klinefelter syndrome

3. Turner syndrome

4. Wolfram syndrome

5. Friedreich ataxia

6. Huntington chorea

7. Laurence-Moon-Biedl syndrome

8. Myotonic dystrophy

9. Porphyria

10. Prader-Willi syndrome

11. Others

IV. Gestational diabetes mellitus

Patients with any form of diabetes may require insulin treatment at some stage of their disease. Such use of insulin does not, of itself, classify the patient. 
onset or first recognition during pregnancy. Although most cases resolve with delivery, the definition applied whether or not the condition persisted after pregnancy and did not exclude the possibility that unrecognized glucose intolerance may have antedated or begun concomitantly with the pregnancy. This definition facilitated a uniform strategy for detection and classification of GDM, but its limitations were recognized for many years. As the ongoing epidemic of obesity and diabetes has led to more type 2 diabetes in women of childbearing age, the number of pregnant women with undiagnosed type 2 diabetes has increased.

After deliberations in 2008-2009, the International Association of Diabetes and Pregnancy Study Groups (IADPSG), an international consensus group with representatives from multiple obstetrical and diabetes organizations, including the American Diabetes Association (ADA), recommended that high-risk women found to have diabetes at their initial prenatal visit, using standard criteria (Table 3), receive a diagnosis of overt, not gestational, diabetes. Approximately $7 \%$ of all pregnancies (ranging from 1 to $14 \%$, depending on the population studied and the diagnostic tests employed) are complicated by GDM, resulting in more than 200,000 cases annually.

\section{CATEGORIES OF INCREASED RISK FOR DIA BETES-In 1997 and} 2003, the Expert Committee on Diagnosis and Classification of Diabetes Mellitus $(1,2)$ recognized an intermediate group of individuals whose glucose levels do not meet criteria for diabetes, yet are higher than those considered normal. These people were defined as having impaired fasting glucose (IFG) [fasting plasma glucose (FPG) levels $100 \mathrm{mg} / \mathrm{dl}(5.6 \mathrm{mmol} / \mathrm{l})$ to $125 \mathrm{mg} / \mathrm{dl}$ (6.9 mmol/1)], or impaired glucose tolerance (IGT) [2-h values in the oral glucose tolerance test (OGTT) of $140 \mathrm{mg} / \mathrm{dl}(7.8 \mathrm{mmol} / \mathrm{l})$ to $199 \mathrm{mg} / \mathrm{dl}$ (11.0 mmol/l)].

Individuals with IFG and/or IGT have been referred to as having prediabetes, indicating the relatively high risk for the future development of diabetes. IFG and IGT should not be viewed as clinical entities in their own right but rather risk factors for diabetes as well as cardiovascular disease. They can be observed as intermediate stages in any of the disease processes listed in Table 1. IFG and IGT are associated with obesity (especially abdominal or visceral obesity), dyslipidemia with high triglycerides and/or low HDL cholesterol, and hypertension. Structured lifestyle intervention, aimed at increasing physical activity and producing 5-10\% loss of body weight, and certain pharmacological agents have been demonstrated to prevent or delay the development of diabetes in people with IGT; the potential impact of such interventions to reduce mortality or the incidence of cardiovascular disease has not been demonstrated to date. It should be noted that the 2003 ADA Expert Committee report reduced the lower FPG cut point to define IFG from $110 \mathrm{mg} / \mathrm{dl}(6.1 \mathrm{mmol} / \mathrm{l})$ to $100 \mathrm{mg} / \mathrm{dl}$ $(5.6 \mathrm{mmol} / \mathrm{l})$, in part to ensure that prevalence of IFG was similar to that of IGT. However, the World Health Organization (WHO) and many other diabetes organizations did not adopt this change in the definition of IFG.

As AlC is used more commonly to diagnose diabetes in individuals with risk factors, it will also identify those at higher risk for developing diabetes in the future. When recommending the use of the AlC to diagnose diabetes in its 2009 report, the International Expert Committee (3) stressed the continuum of risk for diabetes with all glycemic measures and did not formally identify an equivalent intermediate category for A1C. The group did note that those with AlC levels above the laboratory "normal" range but below the diagnostic cut point for diabetes (6.0 to $<6.5 \%$ ) are at very high risk of developing diabetes. Indeed, incidence of diabetes in people with $\mathrm{AlC}$ levels in this range is more than 10 times that of people with lower levels (4-7). However, the 6.0 to $<6.5 \%$ range fails to identify a substantial number of patients who have IFG and/or IGT. Prospective studies indicate that people within the AlC range of 5.5$6.0 \%$ have a 5 -year cumulative incidence of diabetes that ranges from 12 to $25 \%$ (4-7), which is appreciably (three- to eightfold) higher than incidence in the U.S. population as a whole (8). Analyses of nationally representative data from the National Health and Nutrition Examination Survey (NHANES) indicate that the AlC value that most accurately identifies people with IFG or IGT falls between 5.5 and $6.0 \%$. In addition, linear regression analyses of these data indicate that among the nondiabetic adult population, an FPG of $110 \mathrm{mg} / \mathrm{dl}(6.1 \mathrm{mmol} / \mathrm{l})$ corresponds to an A1C of $5.6 \%$, while an FPG of 100 $\mathrm{mg} / \mathrm{dl}(5.6 \mathrm{mmol} / \mathrm{l})$ corresponds to an AlC of $5.4 \%$ (R.T. Ackerman, personal communication). Finally, evidence from the Diabetes Prevention Program (DPP), wherein the mean AlC was 5.9\% (SD $0.5 \%$ ), indicates that preventive interventions are effective in groups of people with A1C levels both below and above 5.9\% (9). For these reasons, the most appropriate $\mathrm{AlC}$ level above which to initiate preventive interventions is likely to be somewhere in the range of 5.5-6\%.

As was the case with FPG and 2-h PG, defining a lower limit of an intermediate category of AlC is somewhat arbitrary, as the risk of diabetes with any measure or surrogate of glycemia is a continuum, extending well into the normal ranges. To maximize equity and efficiency of preventive interventions, such an AlC cut point should balance the costs of "false negatives" (failing to identify those who are going to develop diabetes) against the costs of "false positives" (falsely identifying and then spending intervention resources on those who were not going to develop diabetes anyway).

As is the case with the glucose measures, several prospective studies that used AlC to predict the progression to diabetes demonstrated a strong, continuous association between A1C and subsequent diabetes. In a systematic review of 44,203 individuals from 16 cohort studies with a follow-up interval averaging 5.6 years (range 2.8-12 years), those with an AlC between 5.5 and $6.0 \%$ had a substantially increased risk of diabetes with 5-year incidences ranging from 9 to $25 \%$. An A1C range of $6.0-6.5 \%$ had a 5 -year risk of developing diabetes between 25 and $50 \%$ and relative risk 20 times higher compared with an A1C of 5.0\% (10). In a community-based study of black and white adults without diabetes, baseline AlC was a stronger predictor of subsequent diabetes and cardiovascular events than was fasting glucose (11). Other analyses suggest that an AlC of $5.7 \%$ is associated with similar diabetes risk to the high-risk participants in the DPP (12). Hence, it is reasonable to consider an AlC range of 5.7-6.4\% as identifying individuals with high risk for future diabetes, to whom the term prediabetes may be applied.

Individuals with an A1C of 5.7-6.4\% should be informed of their increased risk for diabetes as well as cardiovascular disease and counseled about effective strategies, such as weight loss and physical activity, to lower their risks. As with glucose measurements, the continuum of risk is curvilinear, so that as $\mathrm{AlC}$ rises, the risk of diabetes rises disproportionately. 
Accordingly, interventions should be most intensive and follow-up should be particularly vigilant for those with AlC levels above $6.0 \%$, who should be considered to be at very high risk. However, just as an individual with a fasting glucose of $98 \mathrm{mg} / \mathrm{dl}$ ( $5.4 \mathrm{mmol} / \mathrm{l})$ may not be at negligible risk for diabetes, individuals with AlC levels below $5.7 \%$ may still be at risk, depending on level of A1C and presence of other risk factors, such as obesity and family history.

Table 2 summarizes the categories of increased risk for diabetes. Evaluation of patients at risk should incorporate a global risk factor assessment for both diabetes and cardiovascular disease. Screening for and counseling about risk of diabetes should always be in the pragmatic context of the patient's comorbidities, life expectancy, personal capacity to engage in lifestyle change, and overall health goals.

\section{DIAGNOSTIC CRITERIA FOR DIABETES MELLITUS-For deca-} des, the diagnosis of diabetes has been based on glucose criteria, either the FPG or the 75-g OGTT. In 1997, the first Expert Committee on the Diagnosis and Classification of Diabetes Mellitus revised the diagnostic criteria, using the observed association between FPG levels and presence of retinopathy as the key factor with which to identify threshold glucose level. The Committee examined data from three cross-sectional epidemiologic studies that assessed retinopathy with fundus photography or direct ophthalmoscopy and measured glycemia as FPG, 2-h PG, and AlC. These studies demonstrated glycemic levels below which there was little prevalent retinopathy and above which the prevalence of retinopathy increased in an apparently linear fashion. The deciles of the three measures at which retinopathy began to increase were the same for each measure within each population. Moreover, the glycemic values above

Table 2-Categories of increased risk for diabetes (prediabetes)*

FPG $100 \mathrm{mg} / \mathrm{dl}$ (5.6 mmol/l) to $125 \mathrm{mg} / \mathrm{dl}$ (6.9 $\mathrm{mmol} / \mathrm{l})$ [IFG]

2-h PG in the 75-g OGTT $140 \mathrm{mg} / \mathrm{dl}$ (7.8 $\mathrm{mmol} / \mathrm{l})$ to $199 \mathrm{mg} / \mathrm{dl}(11.0 \mathrm{mmol} / \mathrm{l})$ [IGT]

AlC 5.7-6.4\%

*For all three tests, risk is continuous, extending below the lower limit of the range and becoming disproportionately greater at higher ends of the range. which retinopathy increased were similar among the populations. These analyses confirmed the long-standing diagnostic 2-h PG value of $\geq 200 \mathrm{mg} / \mathrm{dl}(11.1 \mathrm{mmol} / \mathrm{l})$. However, the older FPG diagnostic cut point of $140 \mathrm{mg} / \mathrm{dl}(7.8 \mathrm{mmol} / \mathrm{l})$ was noted to identify far fewer individuals with diabetes than the 2-h PG cut point. The FPG diagnostic cut point was reduced to $\geq 126 \mathrm{mg} / \mathrm{dl}(7.0 \mathrm{mmol} / \mathrm{l})$.

AlC is a widely used marker of chronic glycemia, reflecting average blood glucose levels over a 2- to 3-month period of time. The test plays a critical role in the management of the patient with diabetes, since it correlates well with both microvascular and, to a lesser extent, macrovascular complications and is widely used as the standard biomarker for the adequacy of glycemic management. Prior Expert Committees have not recommended use of the $\mathrm{AlC}$ for diagnosis of diabetes, in part due to lack of standardization of the assay. However, AlC assays are now highly standardized so that their results can be uniformly applied both temporally and across populations. In their recent report (3), an International Expert Committee, after an extensive review of both established and emerging epidemiological evidence, recommended the use of the AlC test to diagnose diabetes, with a threshold of $\geq 6.5 \%$, and ADA affirms this decision. The diagnostic A1C cut point of $6.5 \%$ is associated with an inflection point for retinopathy prevalence, as are the diagnostic thresholds for FPG and 2-h PG (3). The diagnostic test should be performed using a method that is certified by the $\mathrm{Na}$ tional Glycohemoglobin Standardization Program (NGSP) and standardized or traceable to the Diabetes Control and Complications Trial reference assay. Point-of-care AlC assays are not sufficiently accurate at this time to use for diagnostic purposes.

There is an inherent logic to using a more chronic versus an acute marker of dysglycemia, particularly since the AlC is already widely familiar to clinicians as a marker of glycemic control. Moreover, the AlC has several advantages to the FPG, including greater convenience, since fasting is not required, evidence to suggest greater preanalytical stability, and less day-to-day perturbations during periods of stress and illness. These advantages, however, must be balanced by greater cost, the limited availability of AlC testing in certain regions of the developing world, and the incomplete correlation between AlC and average glucose in certain individuals. In addition, the AlC can be misleading in patients with certain forms of anemia and hemoglobinopathies, which may also have unique ethnic or geographic distributions. For patients with a hemoglobinopathy but normal red cell turnover, such as sickle cell trait, an AlC assay without interference from abnormal hemoglobins should be used (an updated list is available at http://www.ngsp.org/interf.asp). For conditions with abnormal red cell turnover, such as anemias from hemolysis and iron deficiency, the diagnosis of diabetes must employ glucose criteria exclusively.

The established glucose criteria for the diagnosis of diabetes remain valid. These include the FPG and 2-h PG. Additionally, patients with severe hyperglycemia such as those who present with severe classic hyperglycemic symptoms or hyperglycemic crisis can continue to be diagnosed when a random (or casual) plasma glucose of $\geq 200 \mathrm{mg} / \mathrm{dl}$ ( 11.1 $\mathrm{mmol} / \mathrm{l}$ ) is found. It is likely that in such cases the health care professional would also measure an A1C test as part of the initial assessment of the severity of the diabetes and that it would (in most cases) be above the diagnostic cut point for diabetes. However, in rapidly evolving diabetes, such as the development of type 1 diabetes in some children, AlC may not be significantly elevated despite frank diabetes.

Just as there is less than $100 \%$ concordance between the FPG and 2-h PG tests, there is not full concordance between A1C and either glucose-based test. Analyses of NHANES data indicate that, assuming universal screening of the undiagnosed, the AlC cut point of $\geq 6.5 \%$ identifies one-third fewer cases of undiagnosed diabetes than a fasting glucose cut point of $\geq 126 \mathrm{mg} / \mathrm{dl}$ ( 7.0 mmol/l) (www.cdc.gov/diabetes/pubs/ factsheet11/tables1_2.htm). However, in practice, a large portion of the population with type 2 diabetes remains unaware of their condition. Thus, it is conceivable that the lower sensitivity of A1C at the designated cut point will be offset by the test's greater practicality, and that wider application of a more convenient test (A1C) may actually increase the number of diagnoses made.

Further research is needed to better characterize those patients whose glycemic status might be categorized differently by two different tests (e.g., FPG and 
A1C), obtained in close temporal approximation. Such discordance may arise from measurement variability, change over time, or because AlC, FPG, and postchallenge glucose each measure different physiological processes. In the setting of an elevated A1C but "nondiabetic" FPG, the likelihood of greater postprandial glucose levels or increased glycation rates for a given degree of hyperglycemia may be present. In the opposite scenario (high FPG yet AIC below the diabetes cut point), augmented hepatic glucose production or reduced glycation rates may be present.

As with most diagnostic tests, a test result diagnostic of diabetes should be repeated to rule out laboratory error, unless the diagnosis is clear on clinical grounds, such as a patient with classic symptoms of hyperglycemia or hyperglycemic crisis. It is preferable that the same test be repeated for confirmation, since there will be a greater likelihood of concurrence in this case. For example, if the $\mathrm{AlC}$ is $7.0 \%$ and a repeat result is $6.8 \%$, the diagnosis of diabetes is confirmed. However, there are scenarios in which results of two different tests (e.g., FPG and AlC) are available for the same patient. In this situation, if the two different tests are both above the diagnostic thresholds, the diagnosis of diabetes is confirmed.

On the other hand, when two different tests are available in an individual and the results are discordant, the test whose result is above the diagnostic cut point should be repeated, and the diagnosis is made on the basis of the confirmed test. That is, if a patient meets the diabetes criterion of the AlC (two results $\geq 6.5 \%$ ) but not the FPG $(<126 \mathrm{mg} / \mathrm{dl}$ or 7.0 $\mathrm{mmol} / \mathrm{l}$ ), or vice versa, that person should be considered to have diabetes. Admittedly, in most circumstance the "nondiabetic" test is likely to be in a range very close to the threshold that defines diabetes.

Since there is preanalytic and analytic variability of all the tests, it is also possible that when a test whose result was above the diagnostic threshold is repeated, the second value will be below the diagnostic cut point. This is least likely for AlC, somewhat more likely for FPG, and most likely for the 2-h PG. Barring a laboratory error, such patients are likely to have test results near the margins of the threshold for a diagnosis. The healthcare professional might opt to follow the patient closely and repeat the testing in 3-6 months.

The decision about which test to use to assess a specific patient for diabetes should be at the discretion of the health care professional, taking into account the availability and practicality of testing an individual patient or groups of patients. Perhaps more important than which diagnostic test is used, is that the testing for diabetes be performed when indicated. There is discouraging evidence indicating that many at-risk patients still do not receive adequate testing and counseling for this increasingly common disease, or for its frequently accompanying cardiovascular risk factors. The current diagnostic criteria for diabetes are summarized in Table 3.

\section{Diagnosis of GDM}

GDM carries risks for the mother and neonate. The Hyperglycemia and Adverse Pregnancy Outcomes (HAPO) study (13), a large-scale $(\sim 25,000$ pregnant women) multinational epidemiologic study, demonstrated that risk of adverse maternal, fetal, and neonatal outcomes continuously increased as a function of maternal glycemia at 24-28 weeks, even within ranges previously considered normal for pregnancy. For most complications, there was no threshold for risk. These results have led to careful reconsideration of the diagnostic criteria for GDM. After deliberations in 2008-2009, the IADPSG, an international consensus group with representatives from multiple obstetrical and diabetes organizations, including ADA, developed revised recommendations for diagnosing GDM. The group recommended that all women not known to have diabetes undergo a 75-g OGTT at 24-28 weeks of gestation. Additionally, the group developed diagnostic cut points for the fasting, 1-h, and 2-h plasma glucose measurements that conveyed an odds ratio for adverse outcomes of at least 1.75 compared with women with mean glucose levels in the HAPO study. Current screening and diagnostic strategies, based on the IADPSG statement (14), are outlined in Table 4.

These new criteria will significantly increase the prevalence of GDM, primarily because only one abnormal value, not two, is sufficient to make the diagnosis. The ADA recognizes the anticipated significant increase in the incidence of GDM to be diagnosed by these criteria and is sensitive to concerns about the "medicalization" of pregnancies previously categorized as normal. These diagnostic criteria changes are being made in the context of worrisome worldwide increases in obesity and diabetes rates, with the intent of optimizing gestational outcomes for women and their babies.

Admittedly, there are few data from randomized clinical trials regarding therapeutic interventions in women who will now be diagnosed with GDM based on only one blood glucose value above the specified cut points (in contrast to the older criteria that stipulated at least two

\section{Table 4-Screening for and diagnosis of GDM}

Perform a 75-g OGTT, with plasma glucose measurement fasting and at 1 and $2 \mathrm{~h}$, at 24-28 weeks of gestation in women not previously diagnosed with overt diabetes.

The OGTT should be performed in the morning after an overnight fast of at least $8 \mathrm{~h}$.

The diagnosis of GDM is made when any of the following plasma glucose values are exceeded:

- Fasting: $\geq 92 \mathrm{mg} / \mathrm{dl}(5.1 \mathrm{mmol} / \mathrm{l})$

- $1 \mathrm{~h}: \geq 180 \mathrm{mg} / \mathrm{dl}(10.0 \mathrm{mmol} / \mathrm{l})$

- $2 \mathrm{~h}: \geq 153 \mathrm{mg} / \mathrm{dl}(8.5 \mathrm{mmol} / \mathrm{l})$ 
abnormal values). Expected benefits to their pregnancies and offspring is inferred from intervention trials that focused on women with more mild hyperglycemia than identified using older GDM diagnostic criteria and that found modest benefits $(15,16)$. The frequency of their follow-up and blood glucose monitoring is not yet clear but likely to be less intensive than women diagnosed by the older criteria. Additional well-designed clinical studies are needed to determine the optimal intensity of monitoring and treatment of women with GDM diagnosed by the new criteria (that would not have met the prior definition of GDM). It is important to note that $80-90 \%$ of women in both of the mild GDM studies (whose glucose values overlapped with the thresholds recommended herein) could be managed with lifestyle therapy alone.

\section{References}

1. Expert Committee on the Diagnosis and Classification of Diabetes Mellitus. Report of the Expert Committee on the Diagnosis and Classification of Diabetes Mellitus. Diabetes Care 1997;20:1183-1197

2. Genuth S, Alberti KG, Bennett P, Buse J, DeFronzo R, Kahn R, Kitzmiller J, Knowler WC, Lebovitz H, Lernmark A, Nathan D, Palmer J, Rizza R, Saudek C, Shaw J, Steffes M, Stern M, Tuomilehto J, Zimmet P, Expert Committee on the Diagnosis and Classification of Diabetes Mellitus. Followup report on the diagnosis of diabetes mellitus. Diabetes Care 2003;26:3160 3167

3. International Expert Committee. International Expert Committee report on the role of the AlC assay in the diagnosis of diabetes. Diabetes Care 2009;32:13271334

4. Edelman D, Olsen MK, Dudley TK, Harris AC, Oddone EZ. Utility of hemoglobin Alc in predicting diabetes risk. J Gen Intern Med 2004;19:1175-1180

5. Pradhan AD, Rifai N, Buring JE, Ridker PM. Hemoglobin Alc predicts diabetes but not cardiovascular disease in nondiabetic women. Am J Med 2007;120: 720-727

6. Sato KK, Hayashi T, Harita N, Yoneda T, Nakamura Y, Endo G, Kambe H. Combined measurement of fasting plasma glucose and $\mathrm{AlC}$ is effective for the prediction of type 2 diabetes: the Kansai Healthcare Study. Diabetes Care 2009;32: 644-646

7. Shimazaki T, Kadowaki T, Ohyama Y, Ohe K, Kubota K. Hemoglobin Alc (HbAlc) predicts future drug treatment for diabetes mellitus: a follow-up study using routine clinical data in a Japanese university hospital. Translational Research 2007;149:196-204

8. Geiss LS, Pan L, Cadwell B, Gregg EW, Benjamin SM, Engelgau MM. Changes in incidence of diabetes in U.S. adults, 1997-2003. Am J Prev Med 2006;30: 371-377

9. Knowler WC, Barrett-Connor E, Fowler SE, Hamman RF, Lachin JM, Walker EA, Nathan DM, Diabetes Prevention Program Research Group. Reduction in the incidence of type 2 diabetes with lifestyle intervention or metformin. N Engl J Med 2002;346:393-403

10. Zhang X, Gregg EW, Williamson DF, Barker LE, Thomas W, Bullard KW, Imperatore G, Williams DE, Albright AL. AlC level and future risk of diabetes: a systematic review. Diabetes Care 2010; 33:1665-1673
11. Selvin E, Steffes MW, Zhu H, Matsushita K, Wagenknecht L, Pankow J, Coresh J, Brancati FL. Glycated hemoglobin, diabetes, and cardiovascular risk in nondiabetic adults. N Engl J Med 2010;362: 800-811

12. Ackermann RT, Cheng YJ, Williamson DF, Gregg EW. Identifying adults at high risk for diabetes and cardiovascular disease using hemoglobin Alc National Health and Nutrition Examination Survey 2005-2006. Am J Prev Med 2011;40: 11-17

13. Metzger BE, Lowe LP, Dyer AR, Trimble ER, Chaovarindr U, Coustan DR, Hadden DR, McCance DR, Hod M, McIntyre HD, Oats JJ, Persson B, Rogers MS, Sacks DA. Hyperglycemia and adverse pregnancy outcomes. N Engl J Med 2008;358:19912002

14. Metzger BE, Gabbe SG, Persson B, Buchanan TA, Catalano PA, Damm P, Dyer AR, Leiva A, Hod M, Kitzmiler JL, Lowe LP, McIntyre HD, Oats JJ, Omori Y, Schmidt MI. International Association of Diabetes and Pregnancy Study Groups recommendations on the diagnosis and classification of hyperglycemia in pregnancy. Diabetes Care 2010;33:676-682

15. Landon MB, Spong CY, Thom E, Carpenter MW, Ramin SM, Casey B, Wapner RJ, Varner MW, Rouse DJ, Thorp JM, Jr., Sciscione A, Catalano P, Harper M, Saade G, Lain KY, Sorokin Y, Peaceman AM, Tolosa JE, Anderson GB. A multicenter, randomized trial of treatment for mild gestational diabetes. N Engl J Med 2009;361:1339-1348

16. Crowther CA, Hiller JE, Moss JR, McPhee AJ, Jeffries WS, Robinson JS. Effect of treatment of gestational diabetes mellitus on pregnancy outcomes. N Engl J Med 2005;352:2477-2486 